\title{
Recent e-EVN developments
}

\section{Arpad Szomoru ${ }^{1}$}

Joint Institute for VLBI in Europe

Postbus 2, 7990 AA Dwingeloo, the Netherlands

E-mail: szomoruejive.nI

With the formal start of the EXPReS project in March 2006, the development of e-VLBI in Europe has reached a new phase. In this paper, we will give a short review of the past of the eVLBI project and describe the current efforts in SA1, one of the Specific Service Activities of EXPReS, aimed at establishing a production e-VLBI network.

8th European VLBI Network Symposium

Toruń, Poland

September 26-29, 2006

\footnotetext{
${ }^{1}$ Speaker, on behalf of the many people at JIVE and the EVN who have contributed to the e-VLBI effort
} 


\section{Introduction}

Over the last decade, enormous progress has been made in the area of Information and Communications Technology (ICT). Like many other research communities, radio astronomy has greatly benefited from the availability of cheap, large-capacity storage media, affordable high-performance desktop computers and multi-processor PC clusters. These advances in ICT are rapidly changing the way VLBI is done. Improvements in computing speed, storage capacity and communication bandwidth enable new scientific opportunities in VLBI and will reshape its operational model.

Traditionally, VLBI has used longitudinal tapes for recording at rates of up to $512 \mathrm{Mbps}$. These tapes are sent by mail to the correlator where they are played back. Recently, the EVN has switched to Mark5 units, i.e. PC-based disk recorder systems [1]. This has enabled operations at $1 \mathrm{Gbps}$ and considerably improved recording quality, efficiency and reliability. The obvious next step was to eliminate the transport of magnetic media altogether, by sending the bit-streams over internet links.

Fibre communication networks are ideally suited to the real-time transfer of huge amounts of data over long distances. The adoption of direct fibre connections by e-MERLIN signals the progress that is being made in this area. As large networks are being deployed for the use of research in Europe, and as networks become more flexible, the introduction of a real-time production VLBI system has become a realistic goal to pursue.

e-VLBI does offer some important advantages over current practice:

- No consumables. Removing the need for recording media, and its transport, will constitute a considerable saving of money and effort for the EVN.

- Fast turn-around. A real-time connected-element EVN will deliver data products to the users in a matter of days. This will reduce the long delay between conception of a project and actual research, and make the EVN a more exciting and productive instrument to use.

- Reliability. Network and telescope performance will be monitored continuously; feedback to the stations in the case of problems will be nearly immediate.

- Target-of-Opportunity (ToO) support. e-VLBI will open the possibility of doing rapid follow-up on unexpected transient events such as supernova explosions and gamma-ray bursts.

- The possibility of interfacing to future real-time arrays, such as e-MERLIN, LOFAR or SKA.

- Future bandwidth needs. The use of standard off-the-shelf hardware components ensures that e-VLBI will be able to take full advantage of commercially driven technological improvements. Given that 10-Gbps fibre technology has become 
mainstream and $40 \mathrm{Gbps}$ is under development, e-VLBI holds the promise of virtually unlimited bandwidth.

\section{The proof-of-concept project}

Last year, the EVN and JIVE concluded a proof-of-concept (PoC) programme to explore the feasibility of real-time VLBI using IP-routed networks. This PoC was supported by DANTE (Delivery of Advanced Network Technology to Europe), which operates the GÉANT network a collaboration between European National Research and Education Networks (NRENs) and the European Commission - and SURFnet, the Dutch NREN. Its aim was to connect at least four EVN telescopes to the correlator at JIVE, ultimately at data rates of $1 \mathrm{Gbps}$, and feed these data streams directly into the correlator.

The PoC project established a regular scheme of observing tests in which up to 6 telescopes would participate. In connecting the telescopes, local loops (the "last mile" connection) are a critical item. Throughout the project, more telescopes established high-speed links, and at the time the project finished, the Westerbork (Netherlands), Onsala (Sweden), Torun (Poland) and Jodrell Bank (UK) radio telescopes all had 1 Gbps connections, while the connection to Arecibo (USA) was limited by a 155-Mbps link to mainland USA, the connection to Cambridge (UK) to 128 Mbps by a microwave link between Cambridge and Jodrell Bank. JIVE itself was connected by SURFnet to Netherlight in September 2002, and could make use of 6 links, each capable of carrying $1 \mathrm{Gbps}$, and one dedicated 1-Gbps connection to the Westerbork Synthesis Radio Telescope, located about $30 \mathrm{~km}$ from JIVE.

The PoC firmly demonstrated the feasibility of e-VLBI and was the foundation for the success of the EXPReS project.

\section{EXPReS}

\subsection{Aims}

The EXPReS proposal (EXpress Production Real-time e-VLBI Service) to the European Commission is an I3 proposal (Communication \& Network Development Call) that was ranked first out of 43 proposals and was granted nearly full funding to an amount of 3.9 M€. EXPReS officially started in March of 2006 and is coordinated by JIVE. It is a collaboration of many astronomical institutes and NRENs throughout Europe and, in fact, the rest of the world. One of its main aims, through the Specific Service Activity SA1, is to transform the EVN into a fully operational e-VLBI network. Another Service Activity, SA2, covers partial funding of physically connecting telescopes to high-bandwidth networks (the "last mile" problem).

\subsection{Current connectivity}

Since the end of the PoC, several more telescopes have been connected at high bandwidth. Medicina (IT) came online at $1 \mathrm{Gbps}$ in spring 2006, as did Metsähovi (FI), at $10 \mathrm{Gbps}$, and first tests were conducted to TIGO (Chile). The next few telescopes to be connected, Effelsberg 
(DE) and Yebes (SP), will have 10- and 2.5-Gbps connections, while the telescope of Shanghai Observatory already has a 1-Gbps connection to Shanghai; however the connection from Shanghai to Europe remains uncertain. During 2007, both Jodrell Bank and Onsala are expected to upgrade their connections to $10 \mathrm{Gbps}$, while JIVE is expected to be upgraded to $16 \mathrm{x} 1 \mathrm{Gbps}$.

\subsection{Current status}

One of the very first actions within EXPReS was the establishment of a scientific e-VLBI service. The first open call for scientific e-VLBI observations was issued in February 2006 to the astronomical community and in the first half year of 2006 three science e-VLBI runs were conducted. The first one, an 8-hour run, was completely lost due to various problems, but the two following runs, each 24 hours continuously at $128 \mathrm{Mbps}$, were very successful. Both observations led to papers that have been submitted and presented at this conference.

With the start of EXPReS, technical testing was set on a regular basis, and yielded the first European 6-station 256-Mbps fringes and the first 512-Mbps 3-station fringes.

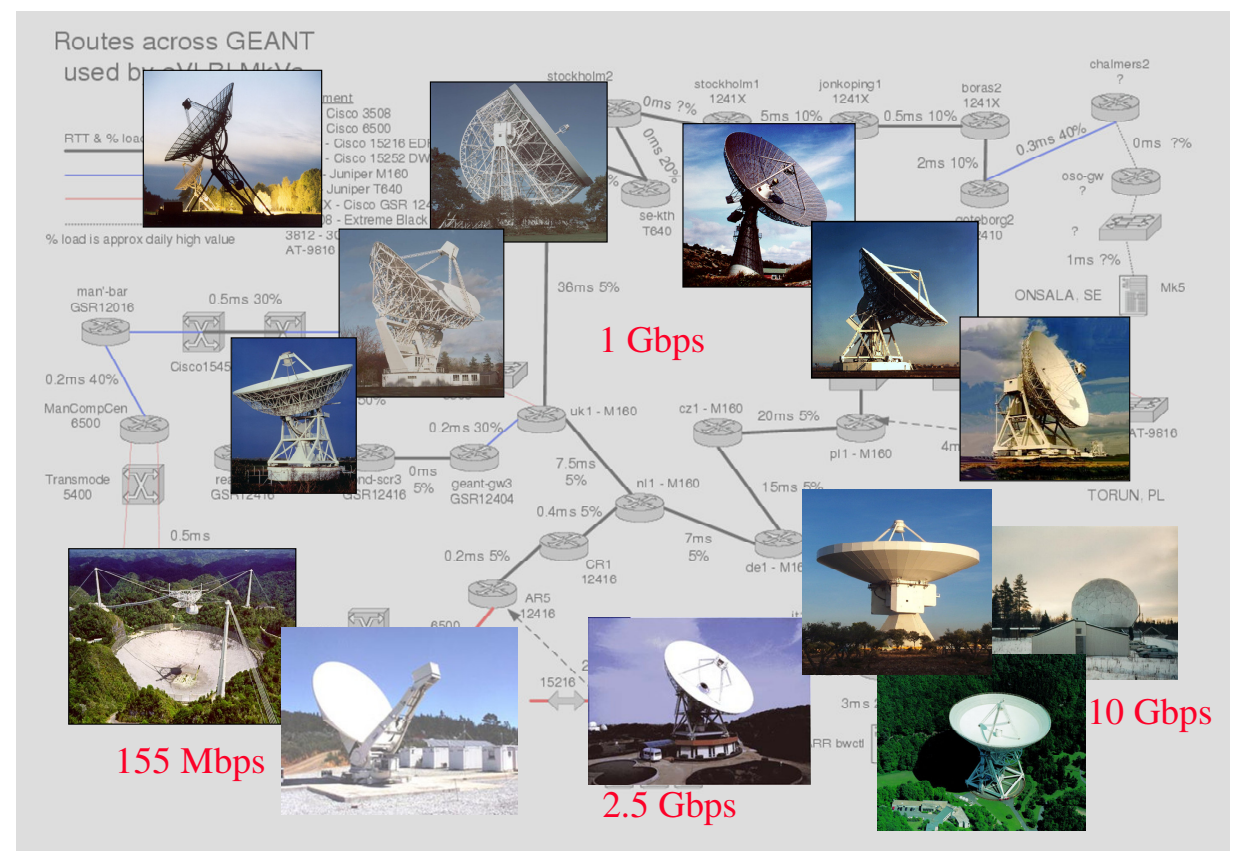

Figure 1: overview of current connectivity to EVN telescopes

\section{New developments}

\subsection{Network upgrades}

Both the SURFnet6 and GÉANT2 networks became operational in spring 2006. Several years ago it was recognized by SURFnet that a traditional IP-switched network would not suffice to combine the extremely large data streams that a few (mostly scientific) applications generate, with an undisrupted internet service to a large number of low-bandwidth users. Because of this, SURFnet6 was designed as a hybrid network, providing both normal IP- 
switched traffic and point-to-point lightpath connections. Likewise, the GÉANT2 network is also fully hybrid. The possibility to make dedicated point-to-point connections is expected to become very important for e-VLBI.

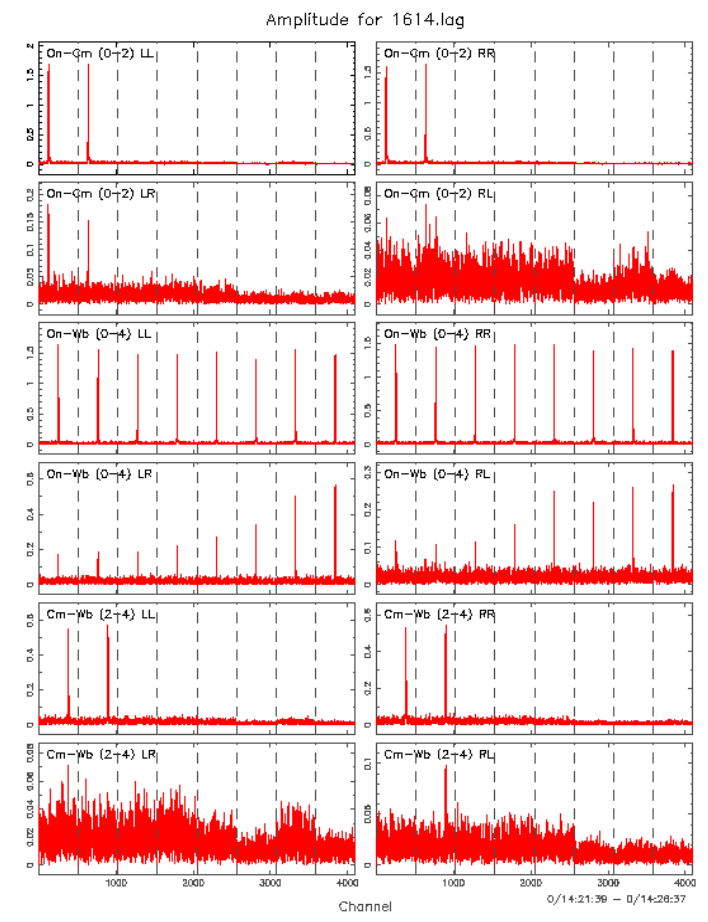

Figure 2: first European three-station 512 Mbps fringes

\section{2 e-VLBI to other continents}

\subsubsection{South America}

The end of the SMART-1 mission on the 3rd of September 2006 provided an opportunity to attempt the first ever e-VLBI transfers from South America. The SMART-1 mission was conceived by ESA as a pathfinder of new technologies, particularly in the area of propulsion and "science reconnaissance". Its controlled impact with the surface of the Moon was to be observed by a network of radio telescopes coordinated by JIVE. The purpose of this was to pinpoint the exact time and location of the impact and to investigate radio-physical effects of wave propagation in close vicinity to the lunar surface. In order to enable rapid analysis of small parts of the data, it was decided to attempt electronic file transfers, as soon after the observations as possible.

Several file transfers were done from TIGO, Chile (one of the few EVN telescopes actually able to observe the impact). During the process of setting up these transfers, we were 
able to determine the topology of the network connection and identify throughput bottlenecks. In spite of the low data rate ( $2 \mathrm{Mbps})$, the transfer yielded good data of the last $20 \mathrm{~ms}$ of the mission within an hour of the observations, and proved to be a very useful exercise.

\subsubsection{China}

The Shanghai Observatory telescope at Seshan is connected to Shanghai at 1 Gbps. At this moment, the equipment at the observatory is not yet capable of making use of this bandwidth, but this will change in the near future. How to connect Shanghai Observatory to Europe at a high data rate is not quite clear yet. Right now, the TEIN2 network provides $622 \mathrm{Mbps}$, and the EC-sponsored ORIENT will increase this to $2.5 \mathrm{Gbps}$. Another option may be to connect Shanghai to JIVE via a dedicated lightpath from Hong Kong to SURFnet. The intention is to include Shanghai Observatory in e-VLBI runs in the beginning of 2007.

\subsubsection{Australia}

Four telescopes of the LBA have been connected to the LBA correlator via 1-Gbps links. Depending on the connectivity between Australia and JIVE, one could in principle observe with the telescopes of the LBA while correlating the data in real time at JIVE. Apart from being an EXPReS deliverable, this would be a powerful demonstrator of the mode in which radio instruments of the future could operate, where the location of the telescopes or correlator has no particular importance and resources are transparently interchangeable. For the time being, the incompatibility of recording and playback hardware (PC-EVN versus Mark5) makes such a demo impossible, however, the possibility of developing a software interface is under investigation.

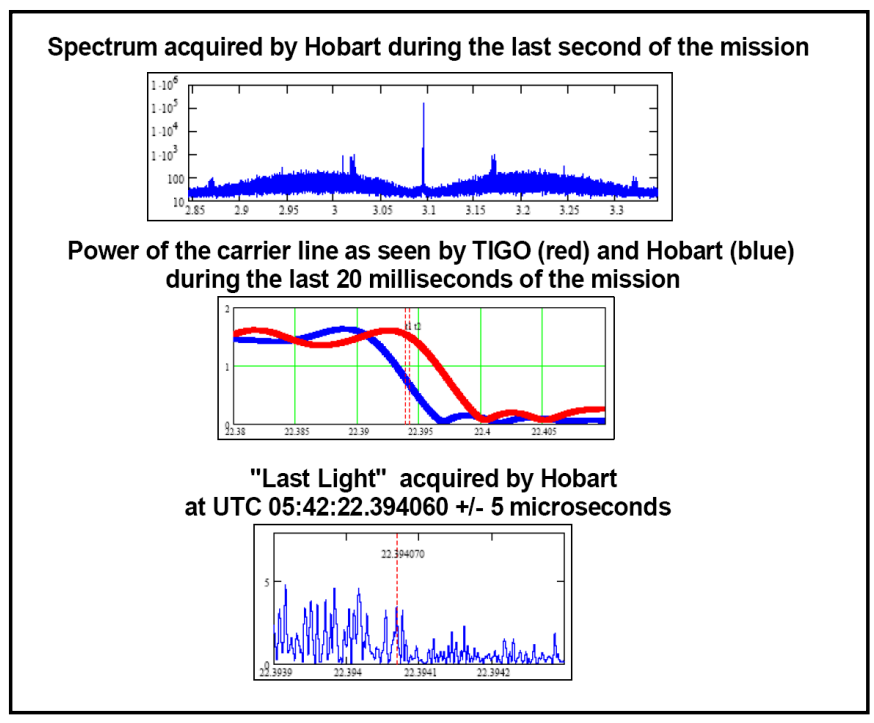

Figure 3: "last light" from SMART-1 


\section{First results of SA1}

\subsection{New positions}

Two software engineers and one e-VLBI postdoc joined the JIVE staff before the summer. A network/linux specialist will start work on 1 December 2006. Likewise, at Jodrell Bank (UK) an electronics engineer will start working shortly on VSI interfaces to e-MERLIN telescopes.

\subsection{Software improvements}

One of the problems of doing e-VLBI is the fact that VLBI systems were originally designed for tape operation. The possibility of doing disk-based, let alone real-time operations, did not come into consideration.

As a rule, experiments are observed and correlated some weeks after the observations. To do this, the system maintains a Reconstituted Observing Time (ROT) clock during playback. For real-time operations, the system had to be modified first to use wall-clock time instead of ROT. Other modifications were made to transparently connect stations to JIVE via the internet, and to let the correlator control computer send commands directly to the Mark5 recording units at the stations, thereby greatly simplifying e-VLBI operations at the correlator.

e-VLBI, by nature, is time-critical. It is essential that results are made available rapidly, that system checks can be made in near real-time and that problems with the system (correlator or stations) are detected and rectified as soon as possible. Instant feedback of status information to the stations is also very important.

A number of new software modules were developed to address these issues. The first to become operational was the Integrating Fringe Display. Fringe display is a powerful tool to monitor the system status, but, in its previous form, was only useful in the case of bright sources, normally fringe finders. This is because a fringe is calculated for every correlated sample. Most of the observing time is spent on target sources, which are often weak and thus undetectable. In the case of ToO observations it would be very useful to be able to make sure one is actually observing anything at all. By integrating over a large number of samples, fringes to very weak sources are made visible. These fringes are also made publicly available through the JIVE website.

Another module, the Data Status Monitor, monitors and interactively displays a large number of system diagnostics like weights, autocorrelations and data rate. This tool is also made available to the stations via a webpage, giving operators an immediate overview of the performance of their telescopes.

An effort is also ongoing to streamline post-processing. This is important to make it possible to rapidly produce maps and diagnostic plots of part of the data, during observations. This web-based tool will only be used at the correlator. 


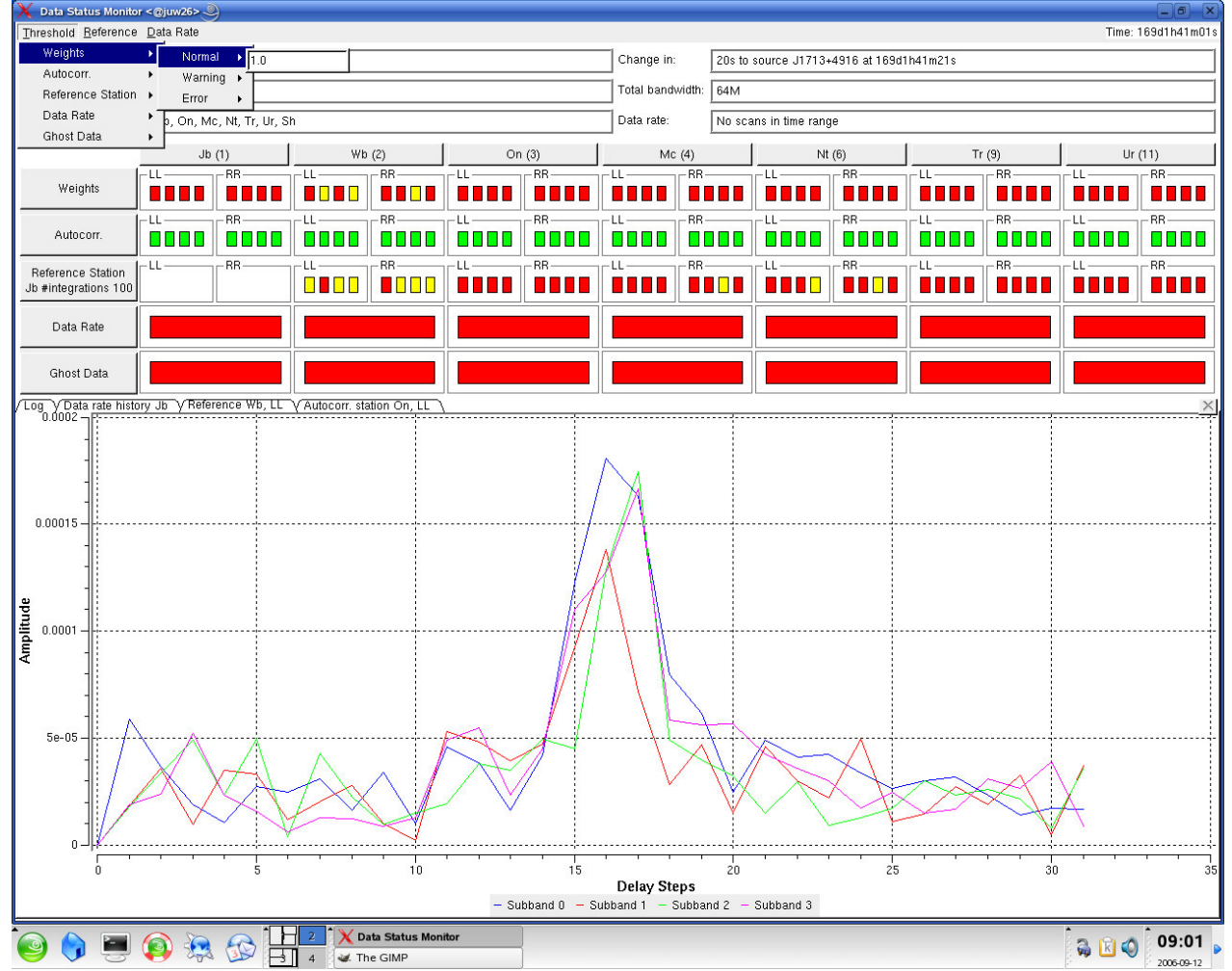

Figure 4: GUI of Data Status Monitor

\subsection{Hardware improvements}

Two heavy-duty Solaris servers were purchased to replace the ageing HP correlator control computer. These machines, equipped with redundant power supplies and dual AMD processors, are meant to be fully and instantly interchangeable, and provide powerful operational and code development platforms. The porting of local and third party software packages to Solaris provided an opportunity to fix and tighten existing code, which has led to considerable improvements and increased robustness of the control system.

An upgrade from Mark5A to Mark5B is currently being investigated. This involves replacing serial links, Mark5 I/O boards and installing so-called Correlator Interface Boards. This upgrade will eventually allow us to phase out the Station Units, which should have a positive effect on the reliability of the correlator. However, a fairly major software effort will be required for this upgrade.

Finally, upgrades to the networking infrastructure are being discussed with SURFnet. JIVE will probably install switching routers in Amsterdam and at JIVE, enabling up to 16 1Gbps lightpaths to be transferred simultaneously to JIVE, while keeping a 10-Gbps lambda for normal IP traffic (transfers from non-lightpath enabled sites and high-bandwidth transfer tests). 


\subsection{Transfer protocols}

At this time, one of the challenges of e-VLBI is to improve the bandwidth from the stations. Although $256 \mathrm{Mbps}$ is usually possible, for the time being only $128 \mathrm{Mbps}$ is guaranteed. The reason is that the TCP protocol, which is being used by the Mark5 units, was designed to prevent congestion; in the case of packet loss, the protocol will back off by reducing transfer rates, and only slowly recover. This fairness, which is what makes the internet work, poses large problems for e-VLBI.

As dedicated lightpaths to the stations become available, this problem will become less important. However, even on dedicated lightpaths without competing traffic, packet loss, caused by the hardware at the endpoints, can occur. Clearly a more aggressive protocol is needed.

At JIVE, research is being done into modified TCP protocols, among others through simulations. An attempt is also being made to implement a home-grown version of Circuit TCP [2], which is a TCP variant specifically designed for dedicated point-to-point circuits. At the University of Manchester, under the ESLEA project, a UDP-based e-VLBI protocol is being developed.

\section{Conclusions}

In this paper we have summarized the past and the current status of European e-VLBI. At this point, e-VLBI has come of age and has become, albeit on a limited scale, an EVN mode of operation in its own right. The investments made possible by the EXPReS project have made it clear that higher data rates with more telescopes are well within reach. In the near future, technological developments are bound to result in an explosive growth of available bandwidth, making $1 \mathrm{Gbps}$ real-time correlation a realistic goal. Availability of more bandwidth will no doubt create demands for yet more bandwidth, and we should look forward to multi-Gbps correlation. The move towards e-EVN is a logical next step, and will put the EVN on the forefront of future developments. We are well on our way to transforming the EVN to a true connected-element interferometer.

\section{References}

[1] A. R. Whitney, New technologies for VLBI, in ASP Conference Series, 308.

[2] A. P. Mudambi, X. Zheng, and M. Veeraraghavan, A Transport Protocol for Dedicated End-toEnd Circuits, in Proc. of ICC 2006, 11-15 June 2006, Istanbul, Turkey.

\section{Acknowledgments}

The European VLBI Network is a joint facility of European, Chinese, South African and other radio astronomy institutes funded by their national research councils. JIVE and the EVN gratefully acknowledge the support they have received in developing European e-VLBI from DANTE, the operator of the pan-European GÉANT2 network, and from the various NRENs, in particular the Dutch SURFnet. 